\title{
A new method for estimating derivatives based on a distribution approach.
}

\author{
Nathalie Verdière* \\ University of Le Havre, LMAH, BP 1123, 76063 Le Havre Cedex, France \\ Lilianne Denis-Vidal \\ University of Sciences and Technologies of Lille, 59650 Villeneuve d'Ascq, France \\ Ghislaine Joly-Blanchard \\ University of Technology of Compiègne, BP 20 529, 60205 Compiègne, France
}

\begin{abstract}
In many applications, the estimation of derivatives has to be done from noisy measured signal. In this paper, an original method based on a distribution approach is presented. Its interest is to report the derivatives on infinitely differentiable functions. Thus, the estimation of the derivatives is done only from the signal. Besides, this method gives some explicit formulae leading to fast calculus. For all these reasons, it is an efficient method in the case of noisy signals as it will be confirmed in several examples.
\end{abstract}

Keywords: Derivatives estimation; Distributions; Test Functions

\section{Introduction}

In many estimation and observation problems [3], [6], [12], derivatives, sometimes of high order, have to be evaluated from a noisy signal given by discrete samples of measurements. It is wellknown that it is an ill-posed problem in numerical analysis and in signal processing and control.

Several approaches for estimating the derivatives of a noisy signal have been proposed in the literature. We can name, for example, methods which consist in approximating the signal by polynomials using least-squares and adding a regularization criteria [2], [7]. A recent one proposed in [1] consists in projecting the signal onto a set of orthogonal functions or modes and approximating the projection by a linear combination of a few of these modes. Fliess et al. have introduced a method based on the local approximation of the signal by a

*Corresponding author 
truncature Taylor expansion and the properties of the Mikusinski field [5], [9]. A study of errors due to the method and the noise on the signal has been treated in $[8]$.

A new method using the distribution theory and the test functions is proposed in this paper. It is based too on the idea of approximating locally the signal by a truncature Taylor expansion but then it uses the properties of the distribution theory. Its first advantage comes from the fact that it allows one to report the derivatives on the test functions which are explicitly known. Thus, the estimation of the derivatives is done only from the signal. Then, this theory introduces naturally integrals which enable us to annihilate a part of the noise on the signal in our estimations. In [9], their formulae are based on the estimation of integrals too which are obtained by integral iterations. An other advantage of our method is that it does not require any statistic knowledge about the noise and gives some explicit expressions leading to fast calculus. Compared to the one proposed by [9], no delay has to be taken into account for improving the estimation of the derivatives. Thus, the derivatives of the signal can be estimated from the transmission of the signal.

This paper is organized as follows. In section 2, the derivative estimation method based on the distribution theory is presented. From some theoretical recall done in section 2.1, the derivative estimation method is deduced and presented at section 2.2 as well as the explicit formulae. In section 3, two types of errors are analyzed: the first one is the error due to the method and which does not consider the noise, the second one is the error due to the noise on the signal. Two inequalities will be given and will serve in the numerical applications, first in giving a criteria for choosing the best test function among others, then for reducing the search of test functions support length. In section 4 , the method is applied on different examples. In order to see how the method works, a simple model based on a sinusoid is used. Then, a pharmacokinetic model is taken again from [12]. In this paper, the estimation of the derivatives specially at $0^{+}$, the right limit of 0 , was important for a parameters estimation problem. Thus, the method is particularly well adapted since it gives explicit formulae at $0^{+}$. Finally, in 4.4, our method is compared with two others. The first one is the method proposed by S. Ibrir and S. Diop in [4]. In this article, they proposed a regularization method based on the B-splines. The second one comes from the article [9] where Fliess et al. analyzed and improved their method by considering barycentric coordinates and by authorizing a delay.

\section{A derivative estimation method based on the distribution theory}

\subsection{Theoretical results}

The signal $x$ is supposed to be an analytic function on $] 0, a[$, equal to zero on ]$-b, 0]$ where $a>0$ or $a=+\infty$ and $b>0$ or $-b=-\infty$. Besides, $x$ is supposed to coincide on $] 0, a[$ with an analytic function $\tilde{x}$ defined on $]-b, a[$. Afterwards, we will say that $x$ verifies the assumption $\mathrm{H}$. 
The signal $x$ can also be associated to a distribution which, in this case, is regular and which operates on the test functions space noticed $\mathcal{D}(]-b, a[)$. These functions are infinitely differentiable and their supports are a compact in ]$-b, a\left[\right.$. The associated distribution is noticed $T_{x}$ and is defined by:

$$
<T_{x}, \varphi>=\int_{]-b, a[} x(t) \varphi(t) d t \text { where } \varphi \in \mathcal{D}(]-b, a[) .
$$

One of the motivations of distributions' use is that they are infinitely differentiable and that the derivation can be reported on the test function. Besides they can take into account the discontinuities, in our case at 0 as it is explicited in the following theorem [10]:

Theorem 1. The Dirac distribution $\delta_{0}$ verifies for all integer $i$ :

$$
<\delta_{0}^{(i)}, \varphi>=(-1)^{i} \varphi^{(i)}(0)
$$

Moreover, if $x$ verifies the assumption $H$, one gets for all $k \in[0, N]$ :

$$
\begin{gathered}
<T_{x}^{(k)}, \varphi>=(-1)^{k}<T_{x}, \varphi^{(k)}>, \\
T_{x}^{(k)}=T_{x^{(k)}}+\sum_{i=0}^{k-1} \sigma_{k-1-i} \delta_{0}^{(i)}, \quad k>0 \\
\text { with } \sigma_{j}=x^{(j)}\left(0^{+}\right)-x^{(j)}\left(0^{-}\right)=x^{(j)}\left(0^{+}\right) \text {for } 0 \leq j \leq N .
\end{gathered}
$$

The notation $0^{+}$(respectively $0^{-}$) designates the right limit at 0 (respectively left limit) of the corresponding functions.

The use of the truncated Taylor expansion for the derivatives estimation is classical (finite difference, etc...). The difference is the way to use it.

Let us consider $x$ a function verifying $\mathrm{H}$ and $\left.t_{l} \in\right] 0, a\left[\right.$ or $t_{l}=0^{+}$for the right limit at 0 .

Let $D\left(t_{l}, R\right)$ defined for all $R$ by $\{t \in]-b, a\left[;\left|t-t_{l}\right|<R\right\}$

Then $\tilde{x}$ is a real analytic function in ] $-b, a[$ and it can be expanded in a power series about the point $t_{l}$ which is its Taylor expansion at $t_{l}$. There exists a number $h, h>0$, such that the series converges in $D\left(t_{l}, h\right)$ and converges uniformly in the compact set $\bar{D}\left(t_{l}, \epsilon\right)$ for all $\epsilon$ such that $0<\epsilon<h$. Let $\epsilon$ be given such that $0<\epsilon<h$. If $0 \in] t_{l}-\epsilon, t_{l}+\epsilon$ [ (respectively $0 \notin\left[t_{l}-\epsilon, t_{l}+\epsilon\right]$ ), the function $x$ is defined by:

$$
x(t)=\left\{\begin{array}{l}
\left.\left.\sum_{n=0}^{+\infty} x^{(n)}\left(t_{l}\right) \frac{\left(t-t_{l}\right)^{n}}{n !}, t \in\right] 0, t_{l}+\epsilon\right] \\
\quad\left(\operatorname{resp} . \quad t \in\left[t_{l}-\epsilon, t_{l}+\epsilon\right]\right) \\
0, t \leq 0,
\end{array}\right.
$$


and the function $x_{N}$ which corresponds to the truncated Taylor expansion

$$
x_{N}(t)=\left\{\begin{array}{l}
\left.\left.\sum_{n=0}^{N} x^{(n)}\left(t_{l}\right) \frac{\left(t-t_{l}\right)^{n}}{n !}, t \in\right] 0, t_{l}+\epsilon\right] \\
\left(\text { resp. } t \in\left[t_{l}-\epsilon, t_{l}+\epsilon\right]\right) \\
0, t \leq 0,
\end{array}\right.
$$

is an approximation of $x(t)$.

Now consider some test functions $\varphi \in \mathcal{D}(]-b, a[)$ whose supports are in $\left[t_{l}-\epsilon, t_{l}+\epsilon\right]$. Afterwards, $\varphi$ is supposed to be positive without any restriction. According to theorem 1, three cases appear. Before giving them, let us introduce $\alpha_{i}=\frac{1}{i !} \int_{\mathcal{V}_{t_{l}}}\left(t-t_{l}\right)^{i} \varphi(t) d t$, where $\mathcal{V}_{t_{l}}$ is specified in each cases. The function test are chosen such that $\alpha_{0} \neq 0$. According to (1), (2) and (4), one gets the three following cases:

- If $0 \notin\left[t_{l}-\epsilon, t_{l}+\epsilon\right], \mathcal{V}_{t_{l}}=\left[t_{l}-\epsilon, t_{l}+\epsilon\right]$ and for $k=1, \ldots, N$,

$$
\sum_{i=k}^{N} x^{(i)}\left(t_{l}\right) \alpha_{i-k}=(-1)^{k} \int_{t_{l}-\epsilon}^{t_{l}+\epsilon} x_{N}(t) \cdot \varphi^{(k)}(t) d t
$$

- If $t_{l}=0^{+}, \mathcal{V}_{t_{l}}=[0, \epsilon]$ and for $k=1, \ldots, N$,

$$
\begin{aligned}
\sum_{i=k}^{N} x^{(i)}\left(0^{+}\right) \alpha_{i-k}+\sum_{i=0}^{k-1}(-1)^{k-1-i} x^{(i)}\left(0^{+}\right) \varphi^{(k-1-i)}(0) \\
=(-1)^{k} \int_{l}^{\epsilon} x_{N}(t) \cdot \varphi^{(k)}(t) d t
\end{aligned}
$$

- If $0 \in] t_{l}-\epsilon, t_{l}+\epsilon\left[, \mathcal{V}_{t_{l}}=\left[0, t_{l}+\epsilon\right]\right.$ and for $k=1, \ldots, N$,

$$
\begin{gathered}
\sum_{i=0}^{k-1}(-1)^{k-1-i} x_{N}^{(i)}\left(0^{+}\right) \varphi^{(k-1-i)}(0)+\sum_{i=k}^{N} x^{(i)}\left(t_{l}\right) \alpha_{i-k} \\
=(-1)^{k} \int_{l}^{t_{l}+\epsilon} x_{N}(t) \varphi^{(k)}(t) d t
\end{gathered}
$$

And in each previous case, one gets:

$$
\sum_{i=0}^{N} x^{(i)}\left(t_{l}\right) \alpha_{i}=\int_{\mathcal{V}_{t_{l}}} x_{N}(t) \cdot \varphi(t) d t
$$

In systems $(5),(6),(7)$ and $(8), x_{N}(t)$ which is an approximation of $x(t)$ is generally an unkown value. In concrete applications, only measured values of $x(t)$ corrupted by noise are available. Afterwards, $y(t)=x(t)+\eta(t)$ where $\eta$ will denote the noise is the measured signal. The aim of the following section will be to give systems depending uniquely of the measured value $y(t)$ and allowing the estimation of the derivatives. 


\subsection{Estimation of the derivatives}

In this section, formulae deduced from (5), (6), (7) and (8) for evaluating derivatives are explicited in substituting $x_{N}(t)$ by $y(t)$. The deduced systems provide us an estimator $C_{\epsilon, N}^{(i)}\left(t_{l}\right)$ of $x^{(i)}\left(t_{l}\right)$ when $0 \notin\left[t_{l}-\epsilon, t_{l}+\epsilon\right]$ (respectively $t_{l}=0^{+}$and $\left.0 \epsilon\right] t_{l}-\epsilon, t_{l}+\epsilon[)$. As previously, the test function $\varphi \in \mathcal{D}(]-b, a[)$ has a support in $\left[t_{l}-\epsilon, t_{l}+\epsilon\right]$. Consider,

$$
A_{\epsilon, \varphi, N}=\left(\begin{array}{cccc}
\alpha_{0} & \alpha_{1} & \ldots & \alpha_{N} \\
0 & \alpha_{0} & \ldots & \alpha_{N-1} \\
\vdots & \ddots & \ldots & \vdots \\
0 & \ldots & \ldots & \alpha_{0}
\end{array}\right)
$$

$B_{\epsilon, \varphi, N}=$

$$
\begin{gathered}
\left(\begin{array}{rllll}
0 & 0 & \ldots & \ldots & 0 \\
\varphi(0) & 0 & \ldots & \ldots & 0 \\
-\varphi^{\prime}(0) & \varphi(0) & 0 & \ldots & 0 \\
\vdots & \ddots & \ddots & \ddots & \vdots \\
(-1)^{N-1} \varphi^{(N-1)}(0) & \ldots & \ldots & \varphi(0) & 0
\end{array}\right), \\
C_{\epsilon, N}\left(t_{l}\right)=\left(C_{\epsilon, N}^{(i)}\left(t_{l}\right)\right)_{0 \leq i \leq N}, X\left(t_{l}\right)=\left(x^{(i)}\left(t_{l}\right)\right)_{0 \leq i \leq N},
\end{gathered}
$$

and finally,

$$
I_{\epsilon, \varphi, x}\left(t_{l}\right)=\left((-1)^{i} \int_{\mathcal{V}_{t_{l}}} x(t) \varphi^{(i)}(t) d t\right)_{0 \leq i \leq N} .
$$

Clearly, matrices $A_{\epsilon, \varphi, N}$ and $B_{\epsilon, \varphi, N}$ are Triangular Toeplitz matrix.

System (5) completed by (8) with $\mathcal{V}_{t_{l}}=\left[t_{l}-\epsilon, t_{l}+\epsilon\right]$ and $\varphi_{1}$ the corresponding test function, becomes

$$
A_{\epsilon, \varphi_{1}, N} X\left(t_{l}\right)=I_{\epsilon, \varphi_{1}, x_{N}}\left(t_{l}\right)
$$

and the estimate $C_{\epsilon, N}\left(t_{l}\right)$ verifies:

$$
A_{\epsilon, \varphi_{1}, N} C_{\epsilon, N}\left(t_{l}\right)=I_{\epsilon, \varphi_{1}, y}\left(t_{l}\right) .
$$

Matrix $A_{\epsilon, \varphi_{1}, N}$ is triangular and $\operatorname{det}\left(A_{\epsilon, \varphi_{1}, N}\right)=\alpha_{0}^{N} \neq 0$.

System (6) completed by (8) with $\mathcal{V}_{t_{l}}=[0, \epsilon]$ and $\varphi_{2}$ the corresponding test function becomes

$$
\left(A_{\epsilon, \varphi_{2}, N}+B_{\epsilon, \varphi_{2}, N}\right) X\left(0^{+}\right)=I_{\epsilon, \varphi_{2}, x_{N}}\left(0^{+}\right)
$$

and the estimate $C_{\epsilon, N}\left(0^{+}\right)$verifies

$$
\left(A_{\epsilon, \varphi_{2}, N}+B_{\epsilon, \varphi_{2}, N}\right) C_{\epsilon, N}\left(0^{+}\right)=I_{\epsilon, \varphi_{2}, y}\left(0^{+}\right) .
$$


Matrix $D_{\epsilon, \varphi_{2}, N}=A_{\epsilon, \varphi_{2}, N}+B_{\epsilon, \varphi_{2}, N}$ is a Toeplitz matrix and has a non-zero determinant with a good choice of test function $\varphi_{2}$.

System (7) completed by (8) with $\mathcal{V}_{t_{l}}=\left[0, t_{l}+\epsilon\right]$ and $\varphi_{3}$ the corresponding test function becomes

$$
A_{\epsilon, \varphi_{3}, N} X\left(t_{l}\right)=I_{\epsilon, \varphi_{3}, x_{N}}\left(t_{l}\right)-B_{\epsilon, \varphi_{3}, N} X\left(0^{+}\right)
$$

and the estimate $C_{\epsilon, N}\left(t_{l}\right)$ verifies:

$$
A_{\epsilon, \varphi_{3}, N} C_{\epsilon, N}\left(t_{l}\right)=I_{\epsilon, \varphi_{3}, y}\left(t_{l}\right)-B_{\epsilon, \varphi_{3}, N} C_{\epsilon, N}\left(0^{+}\right)
$$

where $C_{\epsilon, N}\left(0^{+}\right)$is given by (16). Matrix $A_{\epsilon, \varphi_{3}, N}$ is triangular and $\operatorname{det}\left(A_{\epsilon, \varphi_{3}, N}\right)=$ $\alpha_{0}^{N} \neq 0$.

In all the cases, the estimation $C_{\epsilon, N}^{(i)}\left(t_{l}\right)$ of the derivatives $x^{(i)}\left(t_{l}\right)$ will be done only from the observation $y$.

\section{Error analysis}

Two types of error have to be analyzed. The first one which does not consider the noise is the error due to the method. It permits to know if the problem is well-posed. The second one is the error due to the noise on the signal. A compromise has to be down between these two errors. Indeed, in order to minimize the first one, the test functions support length must be as small as possible. However, it is in opposition for reducing the second one since the noise is better filtered if the integration interval is greater.

Afterwards, the errors will be evaluated in the more usual case that is, the first one when the support of the test function, denoted $\varphi_{1}$, is included in $\left[t_{l}-\epsilon, t_{l}+\epsilon\right]$ and $\left.\left[t_{l}-\epsilon, t_{l}+\epsilon\right] \subset\right] 0, a\left[\right.$. Thus, only the matrix $A_{\epsilon, \varphi_{1}, N}$ is considered. From the studie of these errors, a criteria will be given for choosing the best test function among others. Then a method for reducing the search of the test functions support length will be deduced. Finally, since our estimates are obtained as output of FIR filters with a noisy input, the output signal to noise ratio can be estimated in the case of a zero-mean white Gaussian and will be given at the end of this section.

\subsection{Error due to the method}

In this section, the notations given in sections 2.1 and 2.2 are taken again and the test functions are supposed to be positive. In order to ease the presentation, the proofs of this section are given at the end of this article.

According to the following theorem, the error due to the method can be controlled owing to the choice of the test function and its support length. 
Theorem 2. For all $N \in \mathbb{N}$ by using previous notations, there exist real numbers $S, R$ and $r$ such that for $\epsilon \leq R$ :

$$
\left\|C_{\epsilon, N}\left(t_{l}\right)-X\left(t_{l}\right)\right\|_{1} \leq S\left(\frac{\epsilon}{r}\right)^{N+1} 2 \epsilon \phi(N)
$$

with $\phi(N)=\sum_{i=0}^{N} \phi_{i}$ and $\phi_{i}=\sup _{t \in\left[t_{l}-\epsilon, t_{l}+\epsilon\right]}\left(\left|\varphi_{1}^{(i)}(t)\right|\right)$.

$\phi(N)$ gives a criteria for choosing a test function from a family of them, a similar criteria will be given in section 3.2 .

The following proposition gives the way that the condition number evolves according to the support length of the test function.

Proposition 1. For all $N \in \mathbb{N}$,

$$
1 \leq \chi\left(A_{\epsilon, \varphi_{1}, N}\right) \leq e^{\epsilon}(1+h(\epsilon))
$$

with $\lim _{\epsilon \rightarrow 0} h(\epsilon)=0$

The latter proposition ensures that for an interval length sufficiently small, the condition number of the matrix defined by system (14) is near to one and this for any test functions. Thus, the resolution of this system is a stable problem according to the perturbations of the second member. However, since in the numerical applications the integrals will be estimated by a discrete method and the noise has to be considered, the support length can not be taken as small as possible.

\subsection{Error due to the noise on the signal}

In [5], the authors bring out two kinds of perturbations. The first ones, like the constant perturbations whose amplitude is unknown are said structured and are solutions of a linear homogeneous differential equation. They can be annihilated by linear differential operators and it is, for example, the case of a random noise whose mean is constant and unknown. The second ones are said unstructured and correspond to a high frequency perturbation which can be reduced by low pass filters like iterative integrals. Thus, any noise $\eta(t)$ can be written as $\eta(t)=\eta_{0}(t)+\gamma$ where $\eta_{0}(t)$ is a noise whose mean is equal to zero and $\gamma$ is a constant representing the mean of $\eta(t)$.

The method based on the distribution theory permits to annihilate a part of the structured noises. When the estimation of the derivatives are done with the formulae (14), (16), (18), the measured signal is only in the second member of the equations. According to the integral linearity, noise appears as $\int_{t_{l}-\epsilon}^{t_{l}+\epsilon} \eta(t) \varphi^{(k)}(t)$. Since $\eta(t)=\eta_{0}(t)+\gamma$, one gets

$$
\int_{t_{l}-\epsilon}^{t_{l}+\epsilon} \eta(t) \varphi^{(k)}(t)=\int_{t_{l}-\epsilon}^{t_{l}+\epsilon} \eta_{0}(t) \varphi^{(k)}(t) d t+\gamma \int_{t_{l}-\epsilon}^{t_{l}+\epsilon} \varphi^{(k)}(t) d t .
$$


If $k \geq 1, \int_{t_{l}-\epsilon}^{t_{l}+\epsilon} \varphi^{(k)}(t)=\varphi^{(k-1)}\left(t_{l}+\epsilon\right)-\varphi^{(k-1)}\left(t_{l}-\epsilon\right)$ is equal to zero as soon as $0^{+} \notin\left[t_{l}-\epsilon, t_{l}+\epsilon\right]$ which will be generally our case in the numerical applications. Thus, constant perturbations are annihilated.

On the other hand, if $0^{+} \in\left[t_{l}-\epsilon, t_{l}+\epsilon\right]$ then $\int_{t_{l}-\epsilon}^{t_{l}+\epsilon} \varphi^{(k)}(t)=-\varphi^{(k-1)}\left(0^{+}\right)$. Thus, constant perturbations can be controlled owing to the test function. Furthermore, according to the following inequality, the choice of the test function can permit to attenuate the zero-mean noise effect. Indeed, one gets:

$$
\left\|\left(\int_{t_{l}-\epsilon}^{t_{l}+\epsilon} \eta_{0}(t) \varphi^{(i)}(t) d t\right)_{0 \leq i \leq N}\right\|_{1}^{2} \leq \int_{t_{l}-\epsilon}^{t_{l}+\epsilon}\left(\eta_{0}(t)\right)^{2} d t \times \psi_{\epsilon, \varphi, N}
$$

and

$$
E\left(\int_{t_{l}-\epsilon}^{t_{l}+\epsilon}\left(\eta_{0}(t)\right)^{2} d t \times \psi_{\epsilon, \varphi, N}\right) \leq \int_{t_{l}-\epsilon}^{t_{l}+\epsilon} \operatorname{var}\left(\eta_{0}(t)\right) d t \times \psi_{\epsilon, \varphi, N}
$$

where var denotes the variance, $E(X)$ the expected value of a random variable $X$ and $\psi_{\epsilon, \varphi, N}=\sum_{i=0}^{N} \int_{t_{l}-\epsilon}^{t_{l}+\epsilon}\left|\varphi^{(i)}(t)\right|^{2} d t . \psi_{\epsilon, N, \varphi}$ gives a criteria for choosing a test function among others. Besides, it permits to determine a lower bound for the test functions support length in order to attenuate a part of the noise. This criteria will be tested on the first example of section 4.2 .

Let us return to the first case that is when $\varphi_{1}$ is considered. In the case of a zero-mean white Gaussian, the bias and variance can be evaluated in order to validate the results. Indeed, if $y(t)=x(t)+\eta_{0}(t)$ where $x(t)$ is the non noisy signal and $\eta_{0}$ is a zero-mean white gaussian noise then the signal derivatives estimates are obtained as output of FIR filters with a noisy input. Suppose that $L$ is the number of samples in $[a, b]$ regularly spaced ${ }^{1}$ and the test function used to estimate the derivatives of $x$ at the sampling time $t_{l}(0 \leq l \leq L)$ has its support contained in an interval of the form $\left[t_{l-M}, t_{l+M}\right]$. Each estimated derivative can be expressed as:

$$
C_{\epsilon, N}^{(n)}\left(t_{l}\right)=\int_{-t_{M}}^{t_{M}} \beta(s) y\left(t_{l}-s\right) d s,
$$

where $\beta$ is a linear combination of the test function $\varphi_{1}\left(t_{l}-.\right)$ and its derivatives. If $W_{m}$ are the weights associated with a given numerical integration method and $\beta_{m}=\beta\left(t_{m}\right)$, for each sampling time $t_{l}$, the following expression is computed:

$$
C_{\epsilon, N}^{(n)}\left(t_{l}\right) \approx \tilde{C}_{\epsilon, N}^{(n)}(l)=\sum_{m=-M}^{M} W_{m} \beta_{m} y_{l-m} .
$$

\footnotetext{
${ }^{1}$ Notice that the following reasoning can be done with a non-uniform sampling.
} 
Denote $c_{m}=W_{m} \beta_{m}$ and $x_{l}^{(n)}=x^{(n)}\left(t_{l}\right)$. Thus, the output signal to noise ratio (SNRout) is given by:

$$
S N \text { Rout }=\frac{\frac{1}{L-2 M} \sum_{l=M}^{L-M}\left|x_{l}^{(n)}\right|^{2}}{\sigma^{2} \sum_{m=-M}^{M}\left|c_{m}\right|^{2}}
$$

Concerning the bias, the relative sample mean squared error due to the bias term and to the numerical approximation of the integrals is computed as:

$$
S B R=\frac{\sum_{l=M}^{L-M}\left|x_{l}^{(n)}\right|^{2}}{\sum_{l=M}^{L-M}\left|x_{l}^{(n)}-E_{s}\left(C_{\epsilon, N}^{(n)}(l)\right)\right|^{2}},
$$

where $E_{s}\left(C_{\epsilon, N}^{(n)}(l)\right)$ is the sample means.

In the numerical applications, the SNRout and SBR will be given in the decadic logarithm.

\subsection{Reduction of the support length search}

The estimation of a upper bound of the relative error between $C_{\epsilon, N}\left(t_{l}\right)$ and $X\left(t_{l}\right)$ will allow one to determine the maximal value of the test functions support length. Without noise, one gets:

$$
\frac{\left\|C_{\epsilon, N}\left(t_{l}\right)-X\left(t_{l}\right)\right\|_{2}^{2}}{\left\|X\left(t_{l}\right)\right\|_{2}^{2}} \leq \chi\left(A_{\epsilon, \varphi_{1}, N}\right)^{2} \frac{\left\|I_{\epsilon, \varphi_{1}, x}\left(t_{l}\right)-I_{\epsilon, \varphi_{1}, x_{N}}\left(t_{l}\right)\right\|_{2}^{2}}{\left\|I_{\epsilon, \varphi_{1}, x}\left(t_{l}\right)\right\|_{2}^{2}}
$$

where $\chi\left(A_{\epsilon, \varphi_{1}, N}\right)$ is the condition number of the matrix $A_{\epsilon, \varphi_{1}, N}$. Thus,

$$
\frac{\left\|X\left(t_{l}\right)\right\|_{2}^{2}}{\left\|C_{\epsilon, N}\left(t_{l}\right)-X\left(t_{l}\right)\right\|_{2}^{2}} \geq \frac{\tilde{E}_{\epsilon, \varphi_{1}, N}}{M^{2} \chi\left(A_{\epsilon, \varphi_{1}, N}\right)^{2}}
$$

where $M=\sup _{t \in\left[t_{l}-\epsilon, t_{l}+\epsilon\right]}\left|x^{(N+1)}(t)\right|$ and

$$
\tilde{E}_{\epsilon, \varphi, N}=\frac{\sum_{i=0}^{N}\left(\int_{t_{l}-\epsilon}^{t_{l}+\epsilon} x(s) \varphi^{(i)}(s) d s\right)^{2}}{\sum_{i=0}^{N}\left(\int_{t_{l}-\epsilon}^{t_{l}+\epsilon} \frac{\left(s-t_{l}\right)^{N+1}}{(N+1) !} \varphi^{(i)}(s) d s\right)^{2}}
$$

In order to obtain good numerical results, $\tilde{E}_{\epsilon, \varphi, N}$ should be maximum. $\tilde{E}_{\epsilon, \varphi, N}$ can be put in correspondance with the SBR. Thus, in the same way as previously and since in the numerical applications only the measured signal $y(t)$ will be accessible at discrete samples, $E_{\epsilon, \varphi, N}$ defined by

$$
E_{\epsilon, \varphi, N}=10 \log _{10} \frac{\sum_{i=0}^{N} \sum_{k=l-M}^{l+M}\left|y\left(t_{k}\right) \varphi^{(i)}\left(t_{k}\right)\right|^{2}}{\sum_{i=0}^{N} \sum_{k=l-M}^{l+M}\left|\frac{\left(t_{k}-t_{l}\right)^{N+1}}{(N+1) !} \varphi^{(i)}\left(t_{k}\right)\right|^{2}}
$$

will be used for reducing the search of the support length of the test functions. 


\section{Numerical applications}

In the previous section, the method for estimating the derivatives from the distribution theory have been established as well as two inequalities. The first one depends on $\psi_{\epsilon, \varphi, N}(22)$ and minimizes the error due to the noise. The second one depends on $E_{\epsilon, \varphi, N}$ and has to be maximized. Since $\psi_{\epsilon, \varphi, N}$ does not depend on the signal, it can be used as a criteria for choosing the best test function and as it will be seen in our examples, it can give the minimal value of the test functions support length. Thus, $\psi_{\epsilon, \varphi, N}$ will be analyzed with two test functions in a first subsection. Then, a simple example is treated for testing the formulae presented at section 2 . In this example, (26) will be analyzed and a method for reducing the search of the interval length will be deduced from $\psi_{\epsilon, \varphi, N}$ and $E_{\epsilon, \varphi, N}$. An application in the pharmacokinetic domain will be then treated. And finally, two examples will be used in order to compare this derivative method with two classical ones.

Thereafter, all the integrals are evaluated with the trapezoidal method.

\subsection{Study of $\psi_{\epsilon, \varphi, N}$}

$\psi_{\epsilon, \varphi, N}$ is an expression depending uniquely on the chosen test function, its derivatives and $N$ and it minimizes the error due to the noise. Thus, in choosing correctly $\epsilon, \varphi, N$ for minimizing $\psi_{\epsilon, \varphi, N}$, this choice permits to attenuate the effect of zero-mean noise on the signal.

In this paper, the measured data are supposed to come from an analytic function. When the derivatives are estimated at $t_{l}$, bell-shaped functions centered at $t_{l}$ have been chosen in order to take into account all the informations of the measured signal in the neighborhood of $t_{l} . \psi_{\epsilon, \varphi, N}$ will be evaluated with the two following test functions:

$$
\hat{\varphi}(t)=\left\{\begin{array}{l}
e^{\frac{\epsilon^{2}}{t^{2}-\epsilon^{2}}} \text { if }|t|<\epsilon \\
0 \text { if }|t| \geq \epsilon,
\end{array}\right.
$$

and

$$
\check{\varphi}(t)=\left\{\begin{array}{l}
\cos ^{2}(t) e^{\frac{\epsilon^{2}}{t^{2}-\epsilon^{2}}} \text { if }|t|<\epsilon \\
0 \text { if }|t| \geq \epsilon,
\end{array}\right.
$$

where $[-\epsilon, \epsilon]$ is the support of the test function. A translation allows to obtain an infinitely differentiable function on any interval.

Afterwards, the solid line curve corresponds to $\hat{\varphi}$ and the dashed line curve to $\check{\varphi}$.

Figure 1 (resp. 2) represents $\Psi_{\epsilon, \varphi, 1}$ (resp. $\Psi_{\epsilon, \varphi, 2}$ ). First, one can notice that for attenuating the effect of the noise with the distribution derivative method, the support length of the chosen test functions must be greater than one. Furthermore, the test function $\check{\varphi}$ seems more efficient than $\hat{\varphi}$ for attenuating the noise on the signal. These remarks will be confirmed in the following example. 


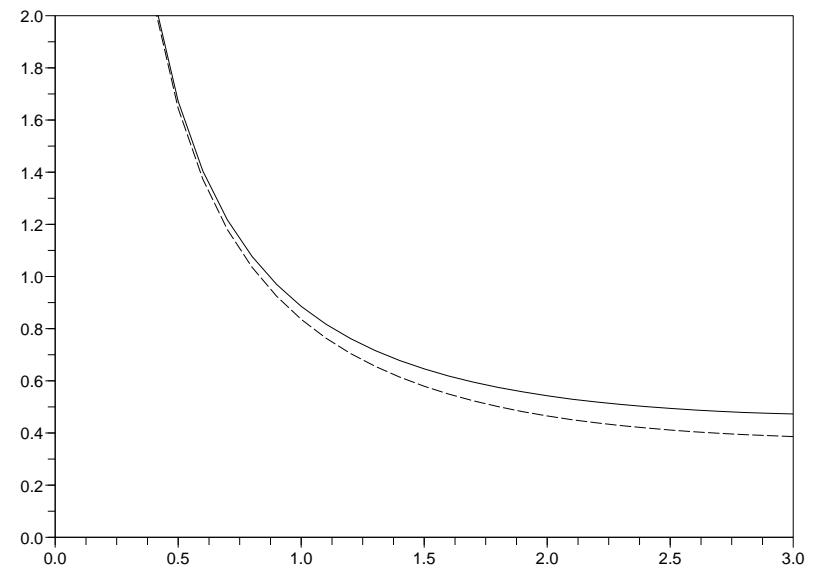

Figure 1: $\Psi_{\epsilon, \varphi, 1}$

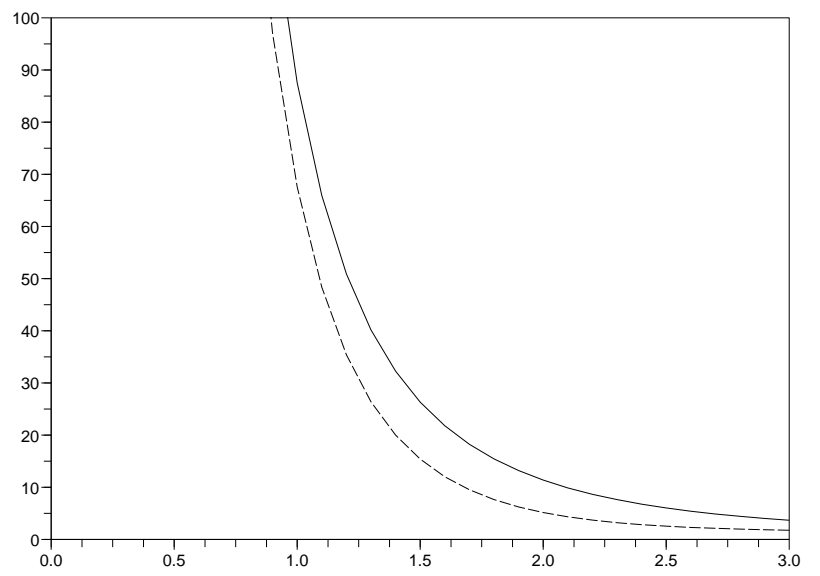

Figure 2: $\Psi_{\epsilon, \varphi, 2}$ 


\subsection{Example 1: Study of $E_{\epsilon, \varphi, N}$}

In this subsection, a very simple model is chosen:

$$
y(t)=\sin \left(\varpi_{0}+\varpi_{1} t\right)+n(t), t \in[0,10]
$$

where $\varpi_{1}=0.8$ is the angular frequency of the signal, $\varpi_{0}=1$ the phase and $n(t)$ is a perturbation. The latter is supposed to follow the normal law with mean-zero and the measures are supposed to be done at each step $h=0.05$, thus there are 200 measured points on the interval $[0,10]$. The signal $y$ is drawn at figure 3. For estimating the first and second order derivatives, the systems

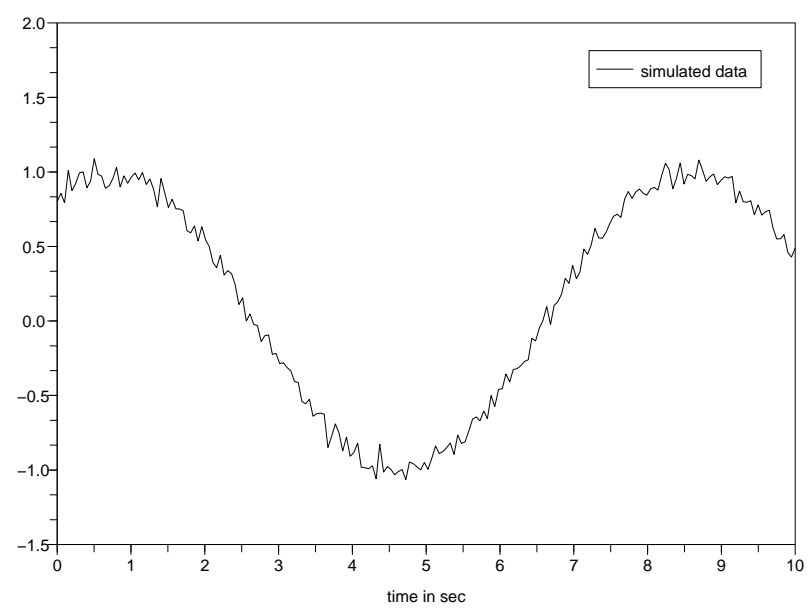

Figure 3: Example 1

(14), (16), (18) are used with $N=1$ (resp. $N=2$ ).

The derivative estimation method presented at section 2 will be tested with the two infinitely differentiable test functions $\hat{\varphi}(27)$ and $\check{\varphi}(28)$.

$E_{\epsilon, \varphi, N}$ given by (26) provides in our examples a criteria for estimating the maximal value of the test functions support length. Figure (4) (resp. (5)) represents $E_{\epsilon, \varphi, 1}$ (resp. $E_{\epsilon, \varphi, 2}$ ). According to the figures, results on the derivative estimates are expected to degrade rapidly as soon as the support length is greater than 3. Furthermore, in this example, for having equivalent results with $\hat{\varphi}$ and $\check{\varphi}$, the support length of $\hat{\varphi}$ must be smaller than $\check{\varphi}$ when it is greater than 1.5. In return, the effect of the noise will be less attenuate. In taken again the remark done at section 4.1, that is the test functions support length must be greater than one, the support length is chosen between 1 and 3 according to the value of $N$. Indeed, when seeking to estimate a highter order derivative, the support length must be greater for better filter the noise.

For $N=1$, the interval length of $\hat{\varphi}$ and $\check{\varphi}$ is equal to 1.5 and their curves coincide. Thus, only the first derivative evaluated with $\check{\varphi}$ is represented at 


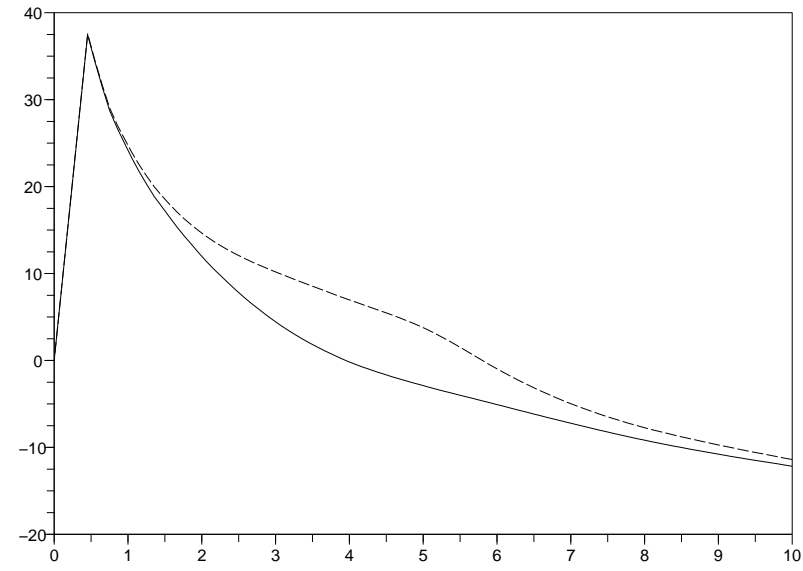

Figure 4: $E_{\epsilon, \varphi, 1}$

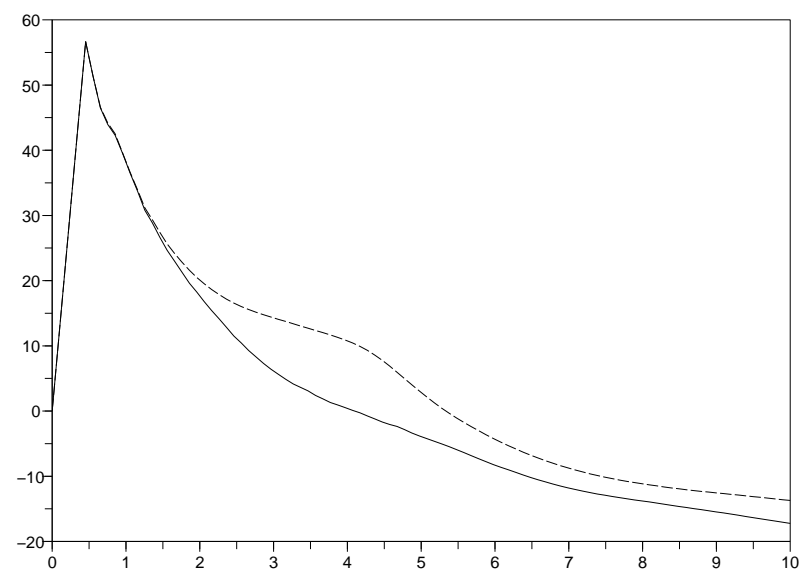

Figure 5: $E_{\epsilon, \varphi, 2}$ 


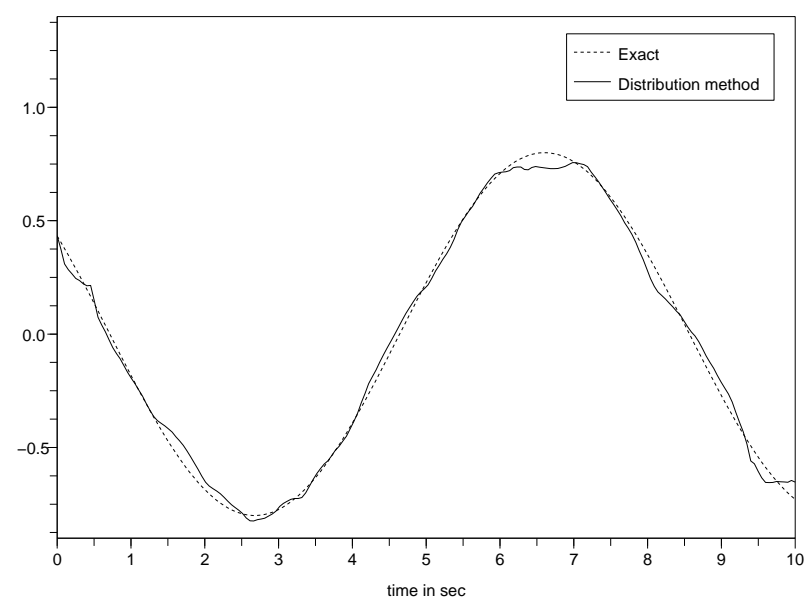

Figure 6: First derivative when $N=1$

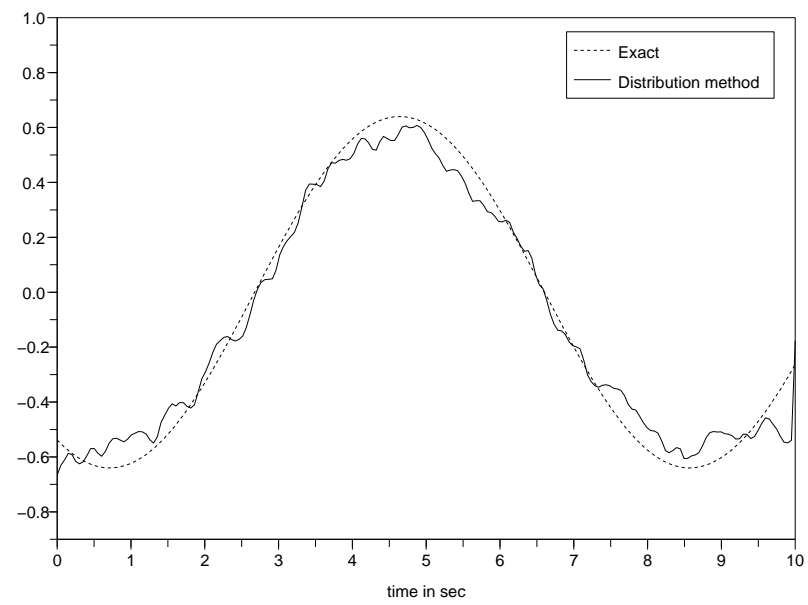

Figure 7: Second derivative evaluated with $\hat{\varphi}$ and $N=2$

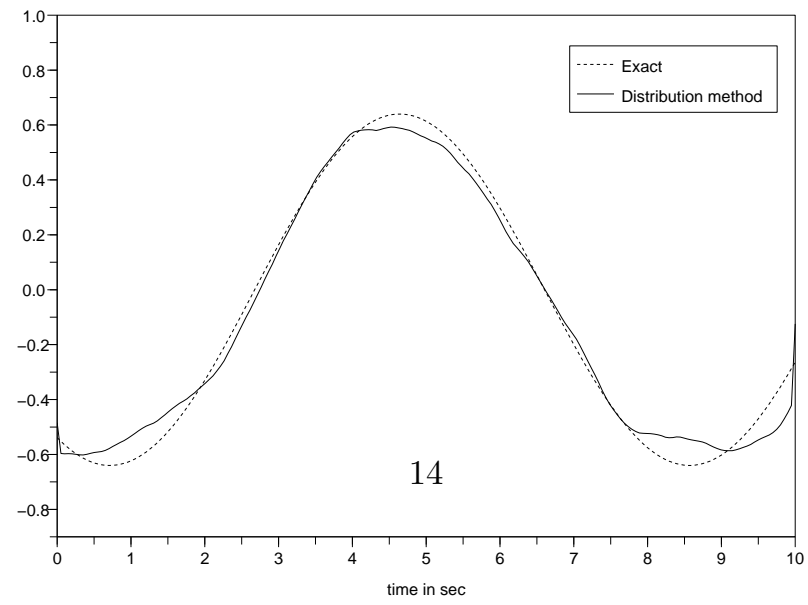

Figure 8: Second derivative evaluated with $\check{\varphi}$ and $N=2$ 
figure 6 . For $N=2$, the interval length for the two test functions is equal to 3 . According to figure 5 , the results for $\check{\varphi}$ should not vary much when the support length vary near 3 and this is confirmed by numerical tests. The second derivative evaluated with $\hat{\varphi}$ is represented at figure 7 . Some irregularities can be noticed for the second derivative unlike the one evaluated with $\check{\varphi}$ (figure 8). For avoiding this phenomenon, the support length of $\hat{\varphi}$ must be chosen greater. The results on the SNRout and the SBR are given at table I. The results confirm that, in this example, the second test function gives better results when $N=2$.

\begin{tabular}{|c|c|c|c|}
\hline & & SNRout & SBR \\
\hline \multirow{2}{*}{$N=1$} & $\hat{\varphi}^{\prime}$ & 36.6 & 24 \\
\cline { 2 - 4 } & $\check{\varphi}^{\prime}$ & 34.1 & 24.01 \\
\hline \multirow{3}{*}{$N=2$} & $\hat{\varphi}^{\prime}$ & 36.4 & 19.58 \\
\cline { 2 - 4 } & $\hat{\varphi}^{\prime \prime}$ & 23.5 & 18.38 \\
\cline { 2 - 4 } & $\check{\varphi}^{\prime}$ & 33.9 & 23.82 \\
\cline { 2 - 4 } & $\check{\varphi}^{\prime \prime}$ & 24.3 & 21.56 \\
\hline
\end{tabular}

One can notice that numerically, the best results are obtained when the order of the truncature Taylor expansion corresponds to the order of the derivative. Furthermore, when the order of the derivative increases, the length of the test function support has to be taken greater.

Afterwards, the function test $\check{\varphi}$ is chosen in the numerical estimation of the derivatives. Besides, the derivatives will be estimated with (14), (16), (18) and the truncature Taylor expansion will correspond to the desired derivative order.

\subsection{Example 2}

The following example (29) is now taken from [12]. Since in this article the estimation of the derivatives near 0 was important for finding the system parameters, the distribution approach is appropriate. This example has been chosen owing to the difficulties of estimating the derivatives, specially the second order derivative whose values are of order $10^{-3}$. Even if they seem negligible, they were important for estimating the system parameters.

$$
\left\{\begin{array}{l}
\dot{x}_{1}=k_{12}\left(x_{2}-x_{1}\right)-k_{v} \frac{x_{1}}{1+x_{1}}, x_{1}(0)=x_{10} \\
\dot{x}_{2}=k_{21}\left(x_{1}-x_{2}\right), x_{2}(0)=0 \\
y=x_{1}
\end{array}\right.
$$

where $x \in \mathbb{R}^{2}$ and $y \in \mathbb{R}$ denote the state variables and the output respectively.

The simulated signal $y$ represented at figure 9 is calculated from the true signal $\bar{y}$ computed with $\bar{\theta}=(0.011,0.02,0.1)$ and with the initial condition $x_{10}=0.625$. The output $y$ is supposed to follow a random law with $\bar{y}$ mean and $(\sigma \bar{y})^{2}$ variance. The coefficient $\sigma$ is computed so that the relative error has a maximum value of 0.05 with an error probability less than 0.003 . 


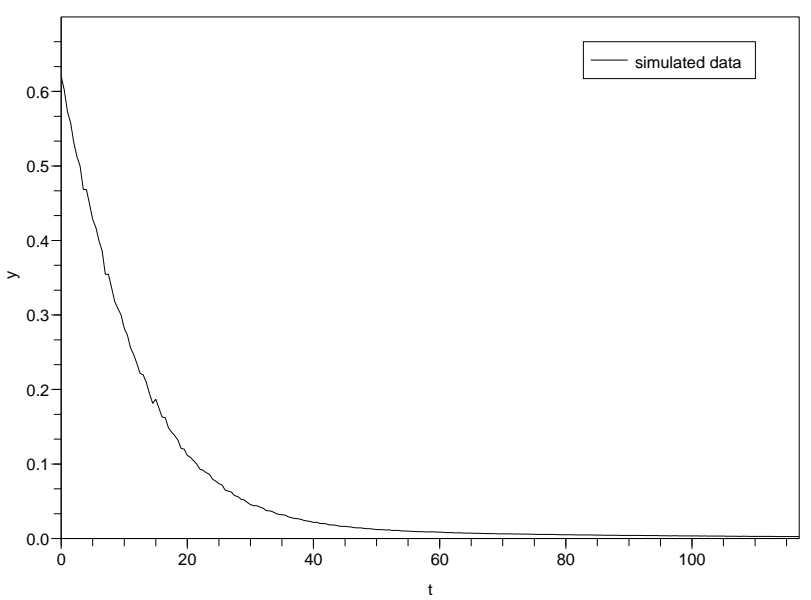

Figure 9: Example 2

The observations are supposed to be done at discrete time $\left(t_{i}\right)_{i=1, \ldots, N}$ on the interval $[0,117]$ with a step equal to 0.5 .

With the same principle as previously, one can deduce from $E_{\epsilon, \breve{\varphi}, 1}$ and $E_{\epsilon, \breve{\varphi}, 2}$ that the intervals length of $\check{\varphi}$ must be less than 30 . The best results are given for an interval length equal to 24 for the first derivative and 29 for the second derivative. The first and second derivatives curves are represented at figure 10 and 11 respectively. As it can be noticed, the results are very satisfactory.

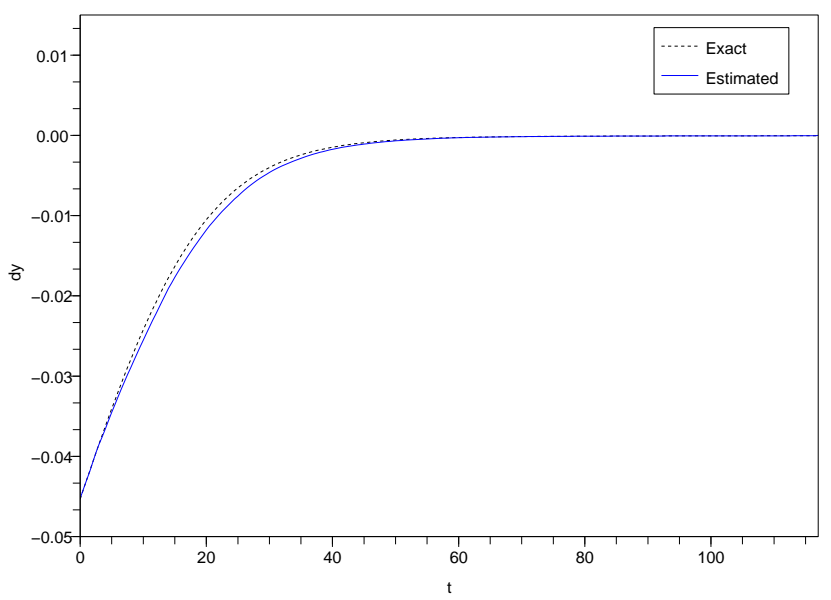

Figure 10: First derivative 


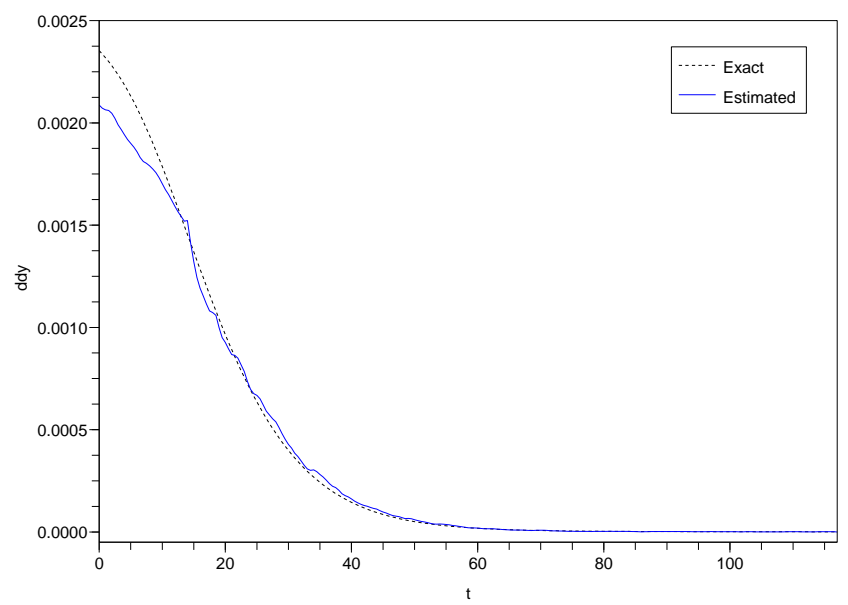

Figure 11: Second derivative

\subsection{Comparison with other methods}

In this section, two other methods for estimating derivatives are considered and compared with the distribution method. The first one is presented in [7] and is based on the B-splines. The second one is proposed by M. Fliess et al. $([5],[9])$. As for the distribution method, these two methods do not require any knowledge of the statistics of measurement uncertainties.

\subsubsection{Example 3}

This example taken from [7] is based on the following noisy signal:

$$
y(t)=\cos (30 t) \sin (t)+\epsilon(t)
$$

and is supposed to be measured for each step $\delta=0.01 \mathrm{~s}$. The function $\epsilon$ follows the normal law whose the variance is unknown. The noisy signal is represented at figure (12). The method proposed in [7] is a regularization method based on the De Boor criteria, 1978, [2] :

$$
J=\frac{1}{n} \sum_{i=1}^{n}\left(y_{i}-\hat{y}_{i}\right)^{2}+\lambda \int_{l}^{t} \hat{y}^{(m)}(s) d s,
$$

which contains a least-squares term symbolizing the connection between the solution of the optimization problem and the measures and a second smoothing term measuring the degree of the solution filtering. The equilibrium between the two distances is controlled by the choice of a parameter $\lambda$ which will be obtained from the optimality condition of GCV criterion (Generalized Cross-Validation criterion) introduced by Craven and Wahba in 1979. De Boor has shown in 1978 that the best results were obtained with a spline function of order $2 \mathrm{~m}$. 


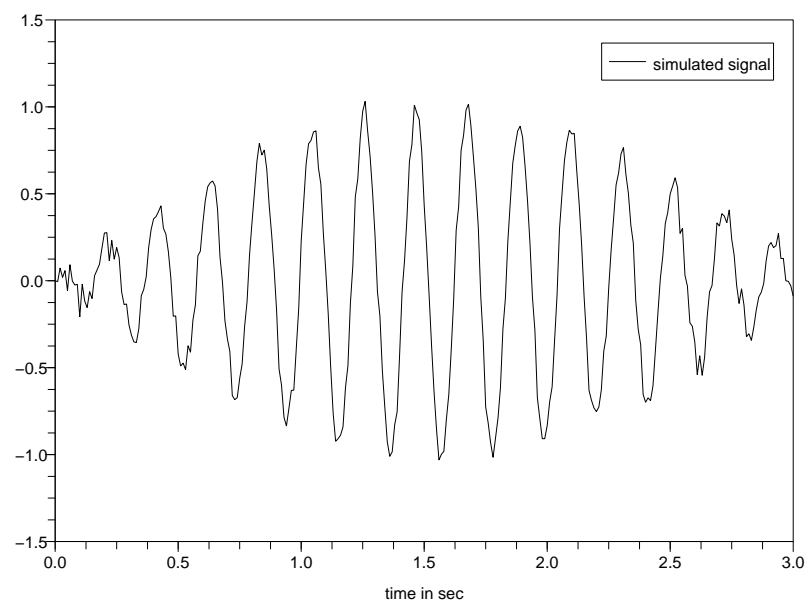

Figure 12: Example 3

In [7], the 3-B-splines have been chosen and an explicit algorithm is deduced. In drawing $E_{\epsilon, \breve{\varphi}, 1}$ and $E_{\epsilon, \breve{\varphi}, 2}$, the support length of $\check{\varphi}$ must be less than 0.3 . The interval on which the integrals are evaluated have a length equal to 0.09 (resp. 0.11 ) for the first derivative (resp. second derivative) which corresponds to 9 points of integration (resp. 11).

The results on the first and second derivatives are represented at figures 13 and 14 respectively.

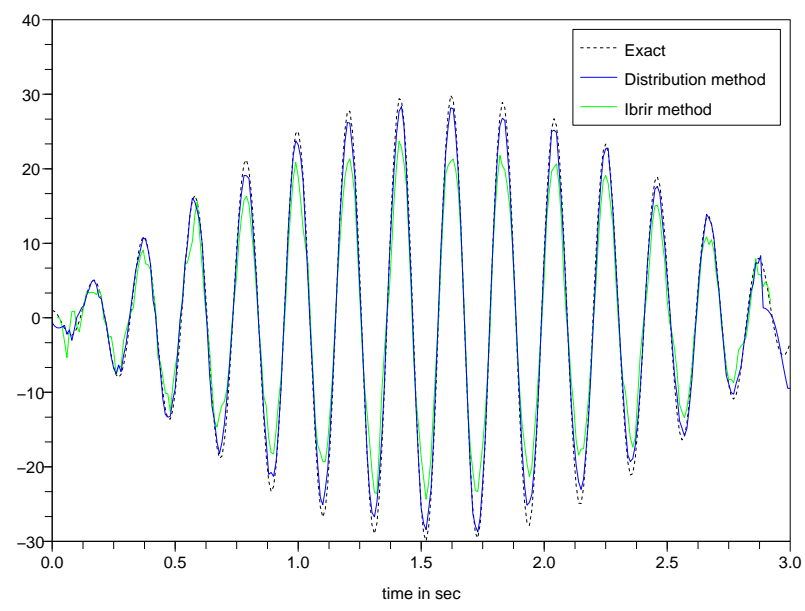

Figure 13: Estimation of the first derivative 


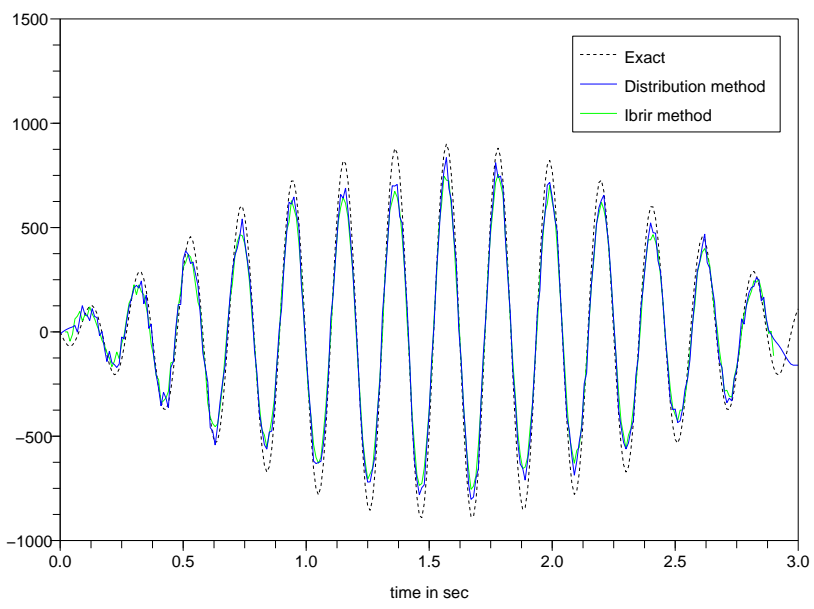

Figure 14: Estimation of the second derivative

\subsubsection{Example 4}

This example comes from [9]. Its starting point is the truncation of the Taylor series expansion of the signal. Instead of working in the distribution space, they operate in the operational calculus domain. Then, in order to have an explicit expression for estimating $\left.\frac{d^{n} x(t)}{d t^{n}}\right|_{t=\tau}$, they use differential elimination and a series of algebraic manipulations. Back in the time domain, they obtain its estimate as an integral operator of the noisy observation within a short time interval $[\tau, \tau+T]$.

The quality of the estimation can be significantly improved by admitting a delay. The delay used in the numerical examples will be $\tau=\zeta_{2} T$ where $\zeta_{2}$ is the largest root of $P_{2}^{\{\kappa, \mu\}}$ which is the third Jacobi orthogonal polynomial. The parameters $\kappa$ and $\mu$ will be given afterwards.

Let $y(t)=x(t)+\varpi(t), 0 \leq t \leq 5$ (without unit) be the noisy measurement of the signal

$$
x(t)=\tanh (t-1)+e^{-t / 1.2} \sin (6 t+\pi) \text { with } t \in[0,5]
$$

and which is measured for each $\delta=0.025$. It corresponds to 1000 samples in the interval $[0,5]$. The signal is supposed to be noisy by a zero-mean white gaussian iid sequence. The variance is adjusted in such a way the signal to noise ratio in $\mathrm{dB}$, i.e, $S N R=10 \log _{10}\left(\frac{\sum\left|y_{i}\right|^{2}}{\sum\left|\varpi_{i}\right|^{2}}\right)$, corresponds to $S N R=25 \mathrm{~dB}$ (see figure $15)$.

In [9], the authors test their method with different values of parameters. Moreover, in order to validate their results, they investigate the bias and variance in each simulation.

For $N=1$, the integrals involved in the formulae are estimated with 60 


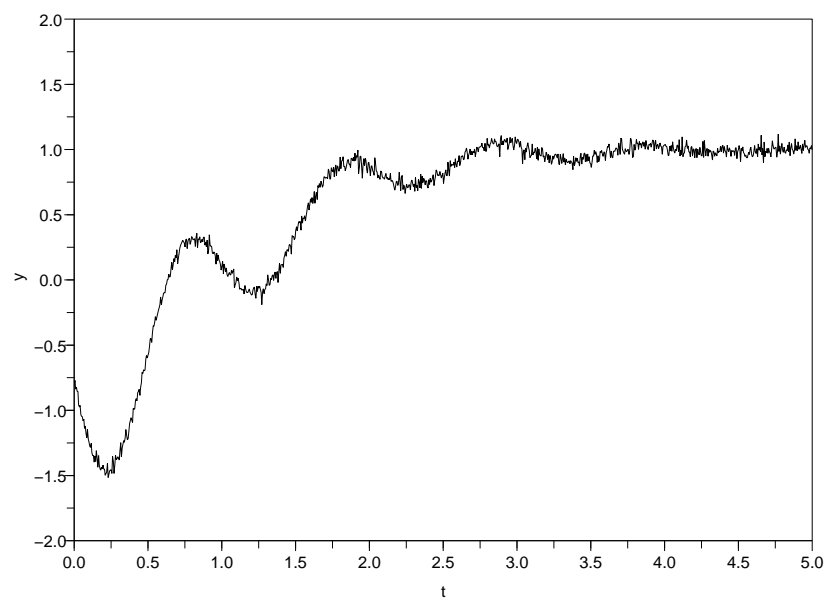

Figure 15: Example 4

points for the two methods. Mboup et al. obtained with $\kappa=\mu=0: \mathrm{SBR}=23.2 \mathrm{~dB}$ and SNRout $=26.5$. For the distribution method, we obtain: $S B R=24.3$ and SNRout $=30.3$. The derivatives are drawn at figure 16 .

For the second derivative, the integrals are calculated with 110 points for the two methods except in the neighborhood of $0^{+}$for the distribution method. Indeed, they are evaluated with 80 points. However, the derivatives in the neighborhood of $0^{+}$are not taken into account for calculating the SBR and the SNRout. The parameters for the Mboup et al. method are $N=2, \kappa=0, \mu=1$ and the SBR and SNRout are equal respectively to $18.1 \mathrm{~dB}$ and $22.6 \mathrm{~dB}$. For the distribution method, we obtain $\mathrm{SBR}=16.2 \mathrm{~dB}$ and $\mathrm{SNR}$ out $=27.2 \mathrm{~dB}$. The obtained figures are drawn at figure 17. In the case of 70 points in the test functions support lenght, the results are: $\mathrm{SBR}=20.3$ and $\mathrm{SNR}$ out $=20.2$.

\section{Conclusion}

In this paper, a new derivative method based on the distribution theory and the test functions is presented. Its first interest comes from the fact that it allows one to report the derivatives on the test functions. Thus, the estimation of the derivatives is done only from the signal. Then, some explicit expressions requiring any knowledge of the statistics of measurement uncertainty are deduced and they lead to fast calculus. Finally, the fact that a regular distribution is a linear integration functional and the use of test functions whose support is chosen adequately permit to annihilate a part of the structured noises. This method was applied and compared with two other methods in order to estimate the derivatives of noisy signals and as it was seen, it gives satisfactory results. 


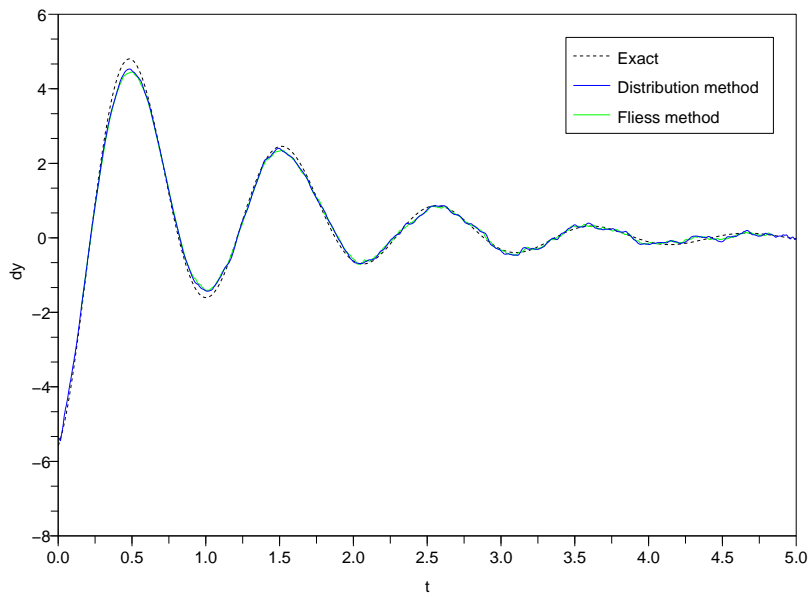

Figure 16: Estimation of the first derivative

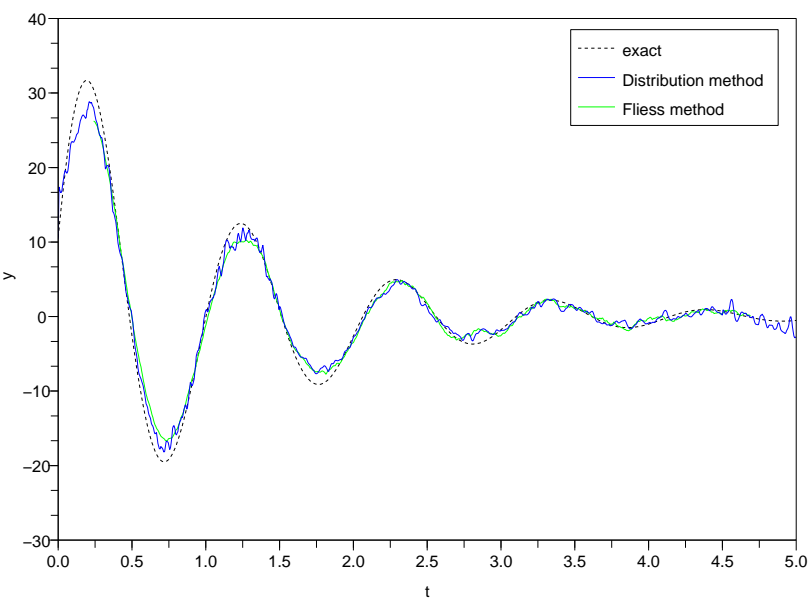

Figure 17: Estimation of the seconde derivative 


\section{Proofs}

Recall that the inverse of an upper triangular Toeplitz invertible matrix is also an upper triangular Toeplitz matrix.

Proof (Proof of theorem 2) From (13) and (14) the following inequality is obtained:

$$
\left\|C_{\epsilon, N}\left(t_{l}\right)-X\left(t_{l}\right)\right\|_{1} \leq\left\|A_{\epsilon, \varphi_{1}, N}^{-1}\right\|_{1}\left\|I_{\epsilon, \varphi, x}\left(t_{l}\right)-I_{\epsilon, \varphi, x_{N}}\left(t_{l}\right)\right\|_{1} .
$$

The two following lemmae will give a priori bounds on $\left\|A_{\epsilon, \varphi_{1}, N}^{-1}\right\|_{1}$ and $\left\|I_{\epsilon, \varphi, x}\left(t_{l}\right)-I_{\epsilon, \varphi, x_{N}}\left(t_{l}\right)\right\|_{1}$.

Lemma 1. There exist real numbers $M$ and $r$ such that:

$$
\left\|I_{\epsilon, \varphi, x}\left(t_{l}\right)-I_{\epsilon, \varphi, x_{N}}\left(t_{l}\right)\right\|_{1} \leq M\left(\frac{\epsilon}{r}\right)^{N+1} 2 \epsilon \phi(N)
$$

with $\phi(N)=\sum_{i=0}^{N} \phi_{i}$ and $\phi_{i}=\sup _{t \in\left[t_{l}-\epsilon, t_{l}+\epsilon\right]}\left(\left|\varphi_{1}^{(i)}(t)\right|\right)$.

Proof (Proof of Lemma 1) Since $x$ is analytic, there exists $\left.\theta_{i, s} \in\right] t_{l}-\epsilon, t_{l}+\epsilon[$ such that

$$
\begin{aligned}
& I_{\epsilon, \varphi, x}\left(t_{l}\right)-I_{\epsilon, \varphi, x_{N}}\left(t_{l}\right)= \\
& \quad\left(\int_{t_{l}-\epsilon}^{t_{l}+\epsilon} \frac{\left(s-t_{l}\right)^{N+1}}{(N+1) !} x^{(N+1)}\left(\theta_{i, s}\right) \varphi_{1}^{(i)}(s) d s\right)_{0 \leq i \leq N} .
\end{aligned}
$$

Furthermore, the analycity of $\mathrm{x}$ on $] 0, a[$ implies the existence of real numbers $\mathrm{M}$ and $\mathrm{r}([11])$ such that for all $t \in\left[t_{l}-h, t_{l}+h\right]$

$$
\frac{\left|x^{(N+1)}(t)\right|}{(N+1) !} \leq \frac{M}{r^{N+1}},
$$

where $\left.\left[t_{l}-\epsilon, t_{l}+\epsilon\right] \subset\left[t_{l}-h, t_{l}+h\right] \subset\right] 0, a[$. Thus lemma 1 is proved.

Lemma 2. There exist real numbers $A_{0}, \ldots, A_{N}$ depending only on the test function $\varphi_{1}$ such that:

$$
\left\|A_{\epsilon, \varphi_{1}, N}^{-1}\right\|_{1} \leq \sum_{i=0}^{i=N} A_{i} \epsilon^{i-1}
$$

with for all $k \in\{1, \ldots, N\}$ :

$-A_{0}=\frac{1}{2 \varphi_{1}(c)}$

$-0<A_{0}=A_{1}<\ldots<A_{N}$ and

$-A_{k}=\frac{A_{0}}{k !}+\frac{\ddot{A_{1}}}{(k-1) !}+. .+\frac{A_{k-1}}{1 !}$. 
Proof (Proof of Lemma 2) The knowledge of the first line or the last column of the matrix $A_{\epsilon, \varphi_{1}, N}^{-1}$ gives all the entries of this matrix. Putting,

$$
A_{\epsilon, \varphi_{1}, N}^{-1}=\left(\begin{array}{cccc}
\beta_{0} & \beta_{1} & \ldots & \beta_{N} \\
0 & \beta_{0} & \ldots & \beta_{N-1} \\
\vdots & \ddots & \ldots & \vdots \\
0 & \ldots & \ldots & \beta_{0}
\end{array}\right)
$$

The last column of this matrix is solution of the system:

$$
\left\{\begin{array}{l}
\beta_{0}=\frac{1}{\alpha_{0}} \\
\beta_{1}=-\frac{\alpha_{1}}{\alpha_{0}} \beta_{0} \\
\ldots \ldots \ldots \ldots \\
\beta_{N}=-\frac{\alpha_{N}}{\alpha_{0}} \beta_{0}-\frac{\alpha_{N-1}}{\alpha_{0}} \beta_{1}-\ldots-\frac{\alpha_{1}}{\alpha_{0}} \beta_{N-1}
\end{array}\right.
$$

Because $\alpha_{0}=\int_{t_{l}-\epsilon}^{t_{l}+\epsilon} \varphi_{1}(t) d t$ and $\varphi_{1}$ is a continuous function there exists $c \epsilon$ ] $t_{l}-\epsilon, t_{l}+\epsilon\left[\right.$ such that $\alpha_{0}=2 \epsilon \varphi_{1}(c)$ and because $\varphi_{1}$ is a positive function $\frac{1}{i !}\left|\int_{t_{l}-\epsilon}^{t_{l}+\epsilon}\left(t-t_{l}\right)^{i} \varphi_{1}(t) d t\right| \leq \frac{\epsilon^{i}}{i !} \alpha_{0}$

This leads to:

$$
\left|\frac{\alpha_{i}}{\alpha_{0}}\right| \leq \frac{\epsilon^{i}}{i !}
$$

By using (38):

$$
\left\{\begin{array}{l}
\left|\beta_{0}\right|=\frac{1}{\alpha_{0}}=\frac{1}{2 \epsilon \varphi_{1}(c)} \\
\left|\beta_{1}\right| \leq \epsilon\left|\beta_{0}\right|
\end{array}\right.
$$

Putting $A_{0}=\frac{1}{2 \varphi_{1}(c)}$ and $A_{1}=\frac{A_{0}}{1 !}$, this gives

$$
\left\{\begin{array}{l}
\left|\beta_{0}\right|=\frac{A_{0}}{\epsilon} \\
\left|\beta_{1}\right| \leq A_{1} \epsilon^{0}
\end{array}\right.
$$

with $\frac{A_{1}}{A_{0}}=1$.

Given any $k \in\{1, \ldots N-1\}$ and suppose that for all $i \in\{1, . ., k-1\}$, $\left|\beta_{i}\right| \leq A_{i} \epsilon^{i-1}$ with $A_{i}$ such that $\frac{A_{i}}{A_{0}}$ is a rational number. According to (38), one gets:

$$
\left|\beta_{k}\right| \leq \frac{\epsilon^{k}}{k !} \frac{A_{0}}{\epsilon}+\ldots+\frac{\epsilon^{i}}{i !} A_{k-i} \epsilon^{k-i-1}+\ldots+\frac{\epsilon}{1 !} A_{k-1} \epsilon^{k-2}
$$

that is,

$$
\left|\beta_{k}\right| \leq A_{k} \epsilon^{k-1}
$$


with $A_{k}=\frac{A_{0}}{k !}+\frac{A_{1}}{(k-1) !}+. .+\frac{A_{k-1}}{1 !}$.

Notice that $\frac{A_{i}}{A_{0}}$ is a rational number because $\frac{A_{1}}{A_{0}}, \ldots, \frac{A_{k-1}}{A_{0}}$ are rational numbers. Furthermore, $A_{0}=\frac{1}{2 \varphi_{1}(c)}>0$ and by simple recurrence $A_{k}>0$ for all $k \in \mathbb{N}$. Since $A_{k}=\frac{A_{k-1}}{1 !}+\ldots+\frac{A_{1}}{(k-1) !}+\frac{A_{0}}{k !}$, one gets $A_{k}>A_{k-1}$ for all $k \in \mathbb{N}^{*}-\{1\}$. Thus, the proof of the lemma 2 is done.

Finally, one can deduce the following lemma.

\section{Lemma 3.}

$$
\left\|C_{\epsilon, N}\left(t_{l}\right)-X\left(t_{l}\right)\right\|_{1} \leq 2\left(\sum_{i=0}^{i=N} A_{i} \epsilon^{i}\right) M\left(\frac{\epsilon}{r}\right)^{N+1} \phi(N) .
$$

For proving the theorem 2, it is sufficient to prove that the series $\sum_{i=0}^{i=+\infty} A_{i} z^{i}$ is convergent when $\epsilon$ is near to zero. This is done by the following lemma.

Lemma 4. The power series $\sum_{i=0}^{i=+\infty} A_{i} z^{i}$ converges for $|z|<R$ with $R>\frac{1}{e-1}$.

Proof (Proof of Lemma 4) By using the lemma 2, one gets

$$
\frac{A_{k+1}}{A_{k}}=\frac{A_{k}}{A_{k}} \frac{1}{1 !}+\ldots+\frac{A_{1}}{A_{k}} \frac{1}{(k-1) !}+\frac{A_{0}}{A_{k}} \frac{1}{k !}
$$

and since $\frac{A_{i}}{A_{k}} \leq 1$ for all $i \in\{0,1, . ., k-1\}$ and $k \in \mathbb{N}^{*}$,

$$
\frac{A_{k+1}}{A_{k}} \leq \frac{1}{1 !}+\ldots+\frac{1}{(k-1) !}+\frac{1}{k !} \leq e-1 \text { for all } k \in \mathbb{N}^{*} .
$$

Hence $\limsup _{k \rightarrow+\infty} \frac{A_{k+1}}{A_{k}} \leq e-1$ and the power series $\sum_{i=0}^{+\infty} A_{i} z^{i}$ has a radius of convergence $R \geq \frac{1}{e-1}$. Finally by using lemmae 3 and 4 , the proof of theorem 2 is done.

Proof (Proof of Proposition 1) By using (38), one gets

$$
\left\|A_{\epsilon, \varphi_{1}, N}\right\|_{1} \leq \sum_{i=0}^{i=N}\left|\alpha_{i}\right| \leq \alpha_{0} \sum_{i=0}^{i=N} \frac{\epsilon^{i}}{i !} \leq \alpha_{0} e^{\epsilon} .
$$

And since there exists $c \in] t_{l}-\epsilon, t_{l}+\epsilon\left[\right.$ such that $\alpha_{0}=2 \epsilon \varphi_{1}(c)$, the following inequalities are obtained:

$$
\alpha_{0} \leq\left\|A_{\epsilon, \varphi_{1}, N}\right\|_{1} \leq 2 \varphi_{1}(c) \epsilon e^{\epsilon}=e^{\epsilon} \alpha_{0}
$$


Besides,

$$
\frac{1}{\alpha_{0}} \leq\left\|A_{\epsilon, \varphi_{1}, N}^{-1}\right\|_{1} \leq \sum_{i=0}^{i=N} A_{i} \epsilon^{i-1}=\frac{1}{\alpha_{0}}+\sum_{i=1}^{i=N} A_{i} \epsilon^{i-1}
$$

and by using (44) and (45), one gets

$$
1 \leq \chi\left(A_{\epsilon, \varphi_{1}, N}\right) \leq e^{\epsilon}+2 \varphi_{1}(c) e^{\epsilon} \sum_{i=1}^{+\infty} A_{i} \epsilon^{i}
$$

By using lemma 4 , the series $\sum_{i=0}^{+\infty} A_{i} \epsilon^{i}$ is convergent when $\epsilon$ is near zero, then $\lim _{\epsilon \rightarrow 0} \sum_{i=1}^{+\infty} A_{i} \epsilon^{i}=0$ and finally $\lim _{\epsilon \rightarrow 0} 2 \varphi_{1}(c) e^{\epsilon} \sum_{i=1}^{+\infty} A_{i} \epsilon^{i}=0$

\section{References}

[1] Ditkowski A, Bhandari A, Sheldon. B. W., "Computing derivatives of noisy signals using orthogonal functions expansions", J. Sci. Comput, 2008, PP. 333-349.

[2] De Boor C., (1878), "A practical Guide to Splines.", New York, Springer.

[3] Diop. S, Grizzle J.W., Morral P.E. and Stefanoupoulou A.G. (1993), "Interpolation and numerical differentiation for observer design." -Proc. Amer. Contr. Conf., Evanston, IL, pp1329-1333.

[4] S. Ibrir, S. Diop (2004), "A numerical procedure for filtering and efficient high-order signal differentiation", Int. J. Appl. Math. Comput. Sci., 2004, Vol. 14, No. 2 -pp201-208.

[5] M. Fliess, M. Mboup, H. Mounier, H. Sira-Ramirez "Questioning some paradigms of signal processing via concret examples", Proc. Summer School: Fast Estimation Method in Automatic Control and Signal Processing, Paris, (2005)

[6] Gauthier J.P., Hammouri H. and Othman S. (1992), " A simple observer for nonlinear systems: Application to bioreactors.", IEEE Trans. Automat. Contr., Vol. 37, No. 6, pp. 675-880.

[7] Ibrir S. and Diop S. (2004), "A numerical procedure for filtering and efficient high-order signal differentiation." Int. J. Appl. Math. Comput. Sci., Vol. 14, No. 2, pp. 201-208.

[8] D. Liu, O. Gibaru and W. Perruquetti. "Error analysis of a new class of derivative estimators for noisy signals", Numerical algorithms, to appear (2011) 
[9] M. Mboup, C. Join, M. Fliess, Numerical differentiation with annihilator in noisy environment, in Numerical Algorithms 50, 4 (2009), 439-467.

[10] W. Rudin," Functional Analysis", McGraw-Hill, 1979.

[11] G.Valiron, "Sur les fonctions analytiques d'une variable réelle", Nouvelles annales de Mathématiques, 5ième série, tome1, p 321-329, 1922.

[12] N. Verdière, L. Denis-Vidal, G. Joly-Blanchard, D. Domurado, "Identifiability and estimation of pharmacokinetic parameters of ligands of macrophage mannose receptor", Int. J. Appl. Math. Comput. Sci, 2005, Vol. 15, No. 4, 101-110. 